\title{
Análisis geológico, estructural y estratigráfico de la ciudad de Macas para la planificación de las zonas de expansión urbana
}

\section{Geological, structural and stratigraphic analyses of the Macas city for the planning of urban expansion areas}

Joao Andres Pasato Jarro. ${ }^{1}$, Gregory Guillermo Cuesta Andrade. ${ }^{2}$, Santiago Nathanael Toapanta Santacruz. ${ }^{3} \&$ Jenny Priscila Granja Carrera. ${ }^{4}$

Recibido: 10-04-2019 / Revisado: 15-05-2019 /Aceptado: 24-06-2019/ Publicado: 15-07-2019

\begin{abstract}
.
DOI: https://doi.org/10.33262/cienciadigital.v3i3.1.701

This research makes a geological, structural and stratigraphic analysis of Macas city, in order to focus planning areas of urban expansion in stable geológicamanete territories. By reviewing several theses and projects in the study area and surrounding areas, and by reconnaissance work in the field, it is observed that the study area is made up of two geological formations:

Mera and Hollín, and a geological unit: Upano. Where a relevant fault passing through the area subandino relief is observed, defining the northern part of the city as the most suitable sector for urban expansion.

Keywords: Geological Lifting, Stratigraphic Lifting - Structural Lifting - Geological Profile - Geological Formations - Geomorphology

1 Escuela Superior Politécnica de Chimborazo, Facultad de Recursos Naturales. Macas, Ecuador. joao.pasato@espoch.edu.ec

${ }^{2}$ Escuela Superior Politécnica de Chimborazo, Facultad de Recursos Naturales. Macas, Ecuador. gregory.cuesta@espoch.edu.ec

3 Escuela Superior Politécnica de Chimborazo, Facultad de Recursos Naturales. Macas, Ecuador. santiago.toapanta@espoch.edu.ec

4 Escuela Superior Politécnica de Chimborazo, Facultad de Recursos Naturales. Macas, Ecuador. jenny.granja@espoch.edu.ec
\end{abstract}




\section{Resumen.}

El presente trabajo realiza un análisis geológico, estructural y estratigráfico de la parroquia Macas, con el objetivo de enfocar la planificación de las zonas de expansión urbana en territorios geológicamanete estables.

Mediante la revisión de varias tesis y proyectos realizados en el área de estudio y zonas aledañas, y mediante trabajos de reconocimiento en campo, se observa que el area de estudio esta constituida por 2 formaciones geológicas: Mera y Hollín, y una unidad geológica: Upano. En donde se observa una falla relevante que pasa por la zona del relieve subandino, definiendo la zona norte de la ciudad como el sector mas adecuado para la expanción urbana.

Palabras claves: Levantamiento Geológico, Levantamiento Estratigráfico - Levantamiento Estructural - Perfil Geológico - Formaciones Geológicas - Geomorfología.

\section{Introducción.}

La inexistencia de información geológica de zonas urbanas no permite un uso adecuado del suelo, generando una planificación deficiente de las zonas de riesgo y zonas de expansión urbana de la parroquia Macas. Por tal razón este trabajo toma en cuenta como información base el mapa geológico regional del Ecuador a escala 1:1`000.000, en donde se observa que el área analizada se encuentra dentro de cinco formaciones geológicas entre ellas la Formación: Mera, Mesa, Napo, Hollín y Tena, de igual manera se utilizó el mapa geológico de Macas a escala 1:100.000; el mapa topográfico de Macas a escala 1:50.000; imágenes satelitales, con la finalidad de tener información útil y detallada.

Esta investigacion realiza un analisis y cotejamiento de varios trabajos de campo y bibliograficos, como el levantamiento geológico, estratigráfico y estructural de la parroquia Macas. Con ayuda de esta información se pretende generar el mapa geológico, estructural y estratigráfico de la parroquia Macas, que sirva como información base para futuros trabajos de ingeniería, y para el ordenamiento territorial de la parroquia, identificando zonas aptas para la expansión urbana y los lugares potencialmente inestables.

\section{Metodología}

Se inicia con la recopilación de información bibliográfica, física y digital preexistente de la zona, como por ejemplo el mapa geológico regional escala 1:250.000, mapa geológico de Macas escala 1:100.000, mapa topográfico del área de estudio a escala 1:50.000 del IGM, Ortofotos, facilitado por el GAD (GAD del Cantón Morona , 2014), fotografías aéreas tomadas con el Drone Phanton 3 Advance propiedad de la Empresa LOWELL.S.A y consultas de Tesis afines a la investigación. 
Se describe la geología Regional de la zona de estudio, donde se detalla las formaciones que afloran en la zona, como tambien la evolución de la Superficie Mera-Upano y el Contexto Estructural de la Cuenca del Oriente donde se habla de las fallas existentes y elevaciones que rodean el área de estudio y su sismicidad.

Para la elaboración del levantamiento geológico, estructural y estratigráfico, primero se prepara la cartografía, donde obtenemos un esquema general de la zona de estudio, en la que se ubica posibles afloramientos, y la descripción geomorfológica de la zona de estudio. Luego se realiza la caracterización geológica de los afloramientos, donde se obtienen datos litológicos, estratigráficos y estructurales mediante la elaboración de fichas de campo en los que constan datos generales del área, perfil del afloramiento, foto y formación a la que pertenece el afloramiento.

Finalmente, con toda la información obtenida tanto bibliografica como de campo, se procede a analizar e interpretar lo resultados para la elaboración de los mapas propuestos y mostrar las zonas mas aptas para la expanción urnaba de la parroquia Macas.

\section{Resultados}

\section{Descripcion de la geologia local}

En la geología local podemos apreciar depósitos de aluviales recientes, formando terrazas constituidas de rocas y arena que se encuentran a lo largo de la cuenca del río Upano.

En la área urbana de Macas se observó dos secuencias de depositación, la capa superior que está formada por coluviales pertenecientes a la formación Mera y la capa inferior que está constituida de rocas tipo lahar, las dos capas antes mencionadas forman la meseta volcanosedimentaria, las mismas que presenta una gradación entre depósitos coluviales y lahares, tienen una matriz limo arenosa y se encuentra delimitada por los ríos Upano y Jurumbaino.

En el sector delimitado por el río Jurumbaino y el flanco oriental del cerro el Quílamo se aprecia afloramientos de limos y limos arenosos depositados en bloques fracturados y erosionados, se determinó el tipo de roca como limolitas.

En el área localizado entre paso lateral y el flanco occidental del cerro el Quílamo se aprecian depósitos de areniscas intercaladas con limolitas y en la parte superior delimitada por el escarpe del Quílamo se encuentran las cuarcitas con potencias variables que van desde los 5 hasta 50 metros, en la parte baja del cerro del Quílamo se observan depósitos coluviales con bloques cuarcíticos con una matriz limo arcillosa

Se puede observar depósitos de rocas metamórficas tipo pizarras y esquistos que se extienden a lo largo del flanco de la cordillera Real Oriental, las mismas que se encuentran en una secuencia rítmica. Tienen potencias variables de 10 hasta 50 metros de espesor. 
Figura 1. Mapa Geológico y Estructural local de la Parroquia Macas

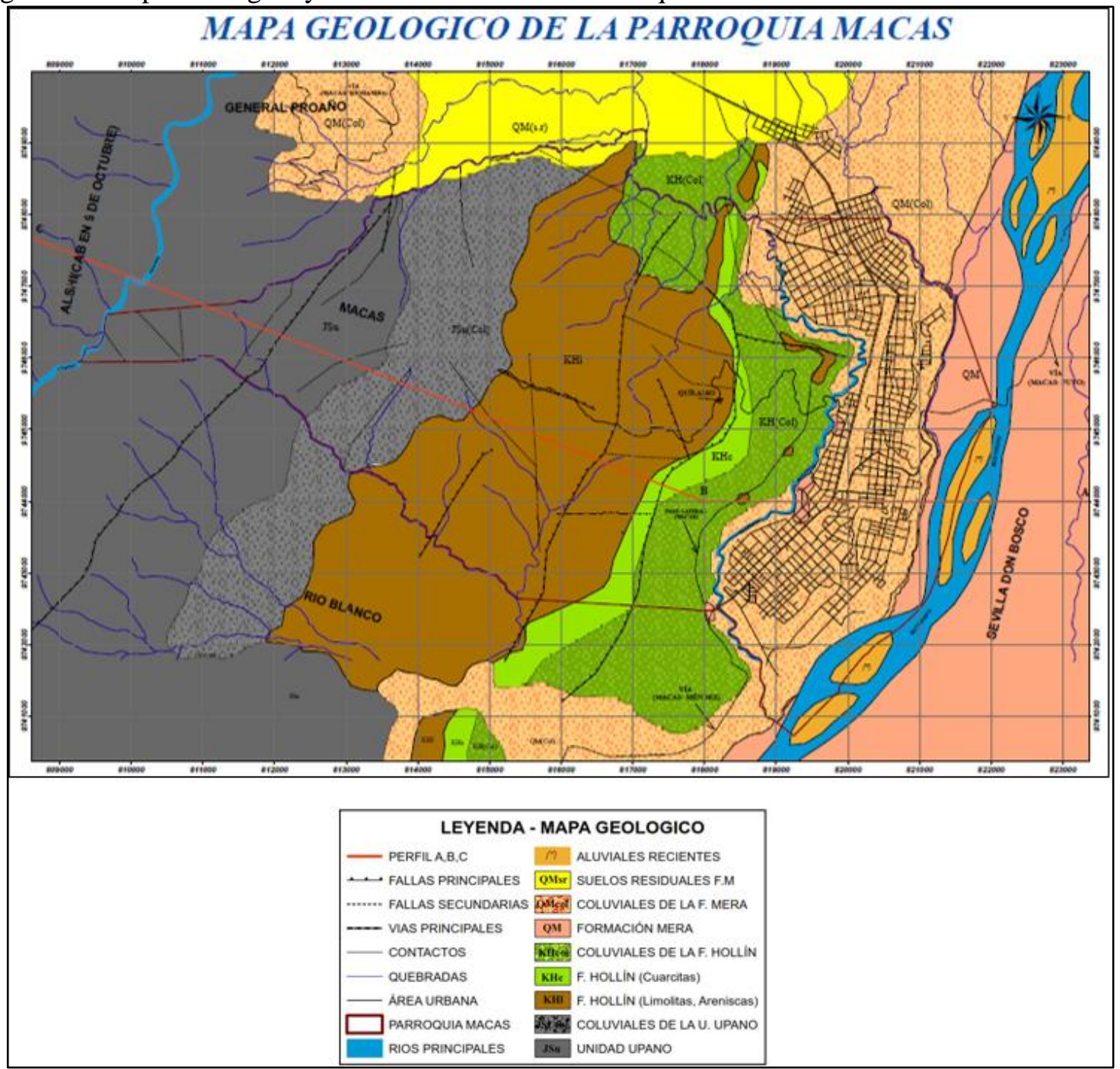

Descripcion de la geologia estructural

En el área de estudio se reconocen dos conjuntos estructurales, la zona tectónica de las escamas al occidente y la llanura del Upano al oriente del área de estudio, el conjunto metamórfico de la cordillera Real se caracteriza por un gran número de direcciones de fallamiento de amplitudes variables. La cuenca de Macas no presenta tectónica en superficie pero se supone la existencia de fallas N-S.

Se evidencia tres fallas geológicas principales que atraviesan en dirección NS. La falla inversa más importante se encuentra ubicada al oeste de la parroquia Macas, producto del levantamiento de la Cordillera Real. Las otras dos fallas inversas se encuentran al este de la Cordillera Real, la una está a lo largo del escarpe del cerro del Quílamo, y la otra atraviesa por la mitad de la formación Hollín con dirección NS.

También se observó fallas menores que cortan perpendicularmente a las fallas principales 
Figura 2. Análisis Estructural de las Secciones A, B, C, D de las Ortofotos del Área de Estudio de la Parroquia Macas

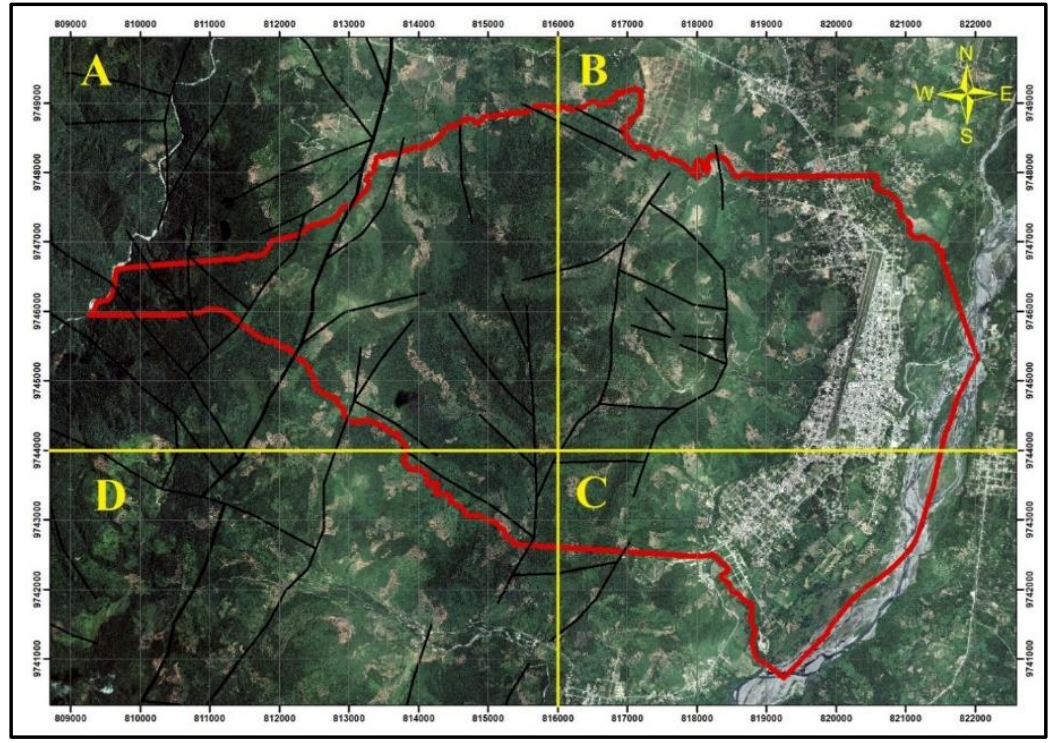

\section{$\underline{\text { Descripcion del perfil estratigrafico }}$}

En el perfil geológico A, B, C de la parroquia Macas, se representa las estructuras y formaciones geológicas en profundidad. Cabe mencionar que es un perfil interpretativo de la geología superficial, construidos con datos reales obtenidos al realizar el levantamiento de campo.

En la figura 2, al Oeste del área de estudio se observa la presencia de una falla regional de tipo inversa, la misma que contiene rocas metamórficas como; esquistos y pizarras pertenecientes a la Unidad Upano, adicionalmente se aprecian dos fallas inversas menores en la misma dirección N-S que cortan en la superficie a la Formación Hollín. En el perfil se puede apreciar dos zonas: la zona Tectónica de las Escamas; donde esta ubicadas todas las fallas principales y secundarias y la Llanura del Upano; no muestra tectónica en superficie, pero no se descartaría fallas y plieguen en profundidad, en esta zona se encuentra el área urbana de la Parroquia Macas.

Figura 3. Perfil Estratigráfico del Corte A-B-C de la Parroquia Macas.

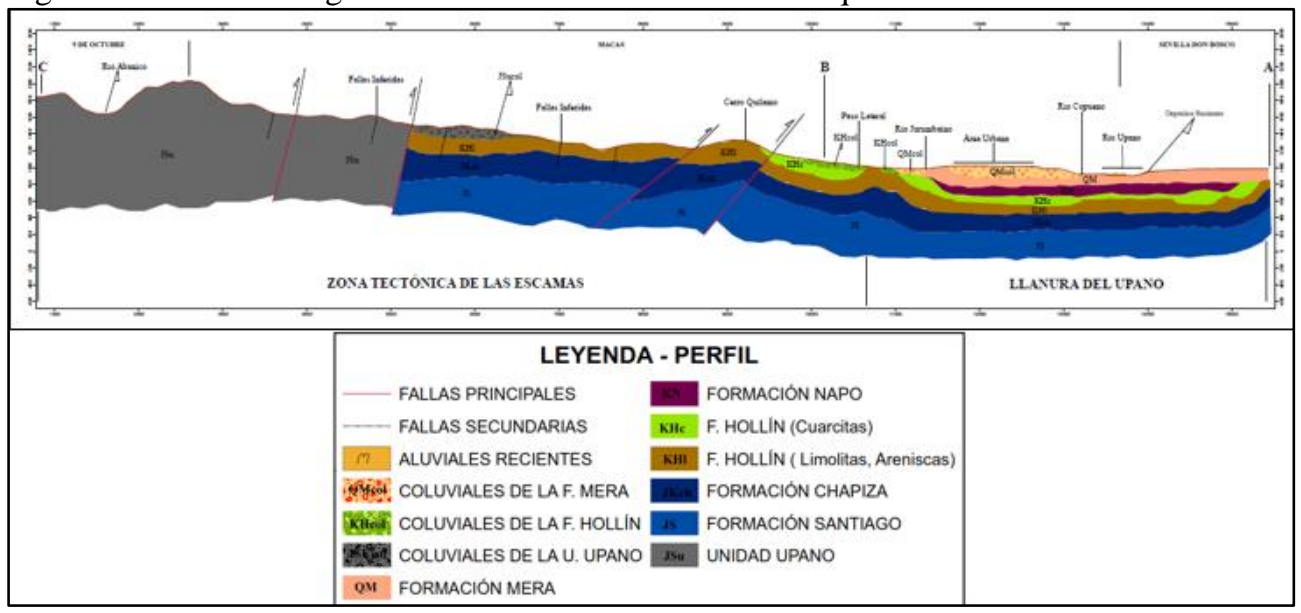




\section{Discusión}

\section{Geologia local}

\section{Aluviales Recientes: (Holoceno)}

Se encuentran a lo largo de la cuenca del rio Upano localizadas en terrazas constituidas de rocas tipos rodados metamórficas, volcánicas, arenas y arcillas. Sus potencias varían de 5 hasta 10 metros.

\section{Formación Mera:(Cuaternario Reciente)}

Depósitos volcanosedimentarios: existen depósitos tipo lahar provenientes del volcán Sangay con clastos angulosos y sub angulosos de diferente granulometría, al ser producto de una avalancha ya sea formada por erupciones volcánicas o por la saturación de agua en los macizos rocosos volcánicos, estos buscan por lo general los causes de los ríos para seguir su trayectoria, están formados por diferentes tipos de rocas y sedmentos, están debajo de los coluviales que se observan en toda el área urbana de la ciudad de Macas.

Depósitos coluviales: se encuentran siguiendo el río Copueno, tienen una matriz compuesta de material limo-arenoso, con clastos que van desde $2 \mathrm{~cm}$ a $20 \mathrm{~cm}$, la litología de los clastos correspondes a lavas andesíticas meteorizadas, los clastos son subangulares y tiene una mala clasificación, también se observan siguiendo el río Jurumbaino y junto al Paso Lateral al sur de la cuidad, está formando la meseta volcanosedimentaria, donde está ubicada la parte urbana de la Parroquia Macas.

Suelo residual: se observó a lo largo de la vía Paso Lateral Norte - Jimbitono, en la parte llana, compuesta por limo arcillas de color café, estos suelos fueron desarrollados por procesos de meteorización de la roca base y de los depósitos coluviales existentes en la zona.

\section{Formación Hollín: (Cretácico Inferior)}

Areniscas: se aprecian areniscas cuarzosas pertenecientes a la formación hollín productos de la meteorización de las cuarcitas de las partes altas de los taludes, se puede visualizar estos tipos de materiales siguiendo el paso lateral y a los márgenes derecho del río jurumbaino.

Cuarcitas: son depósitos de cuarcita levemente metamorfizadas, se encuentran consolidados y formando bloques de gran potencia, constituyen el levantamiento del cerro el Quílamo, se observa al norte del paso lateral y al norte de la parroquia Rio Blanco siguiendo el escarpe del cerro del Quílamo, las fisuras de los bloques cuarcíticos están rellenos de arcillas de color café claro, esta roca está compuesta esencialmente por cuarcita, con pequeñas cantidades de moscovita y ortosa. 
Limolitas: Se observan siguiendo el paso lateral y se encuentran delimitadas por el río Jurumbaino y el flanco del cerro el Quílamo, tienen capas considerables de hasta 40 metro de espesor un una extensión de aproximadamente 4 kilómetros, son materiales de baja plasticidad y se meteorizan con gran facilidad.

Depósitos Coluviales: Se encuentran ubicadas al Nor-Este del cerro del Quílamo en la parte baja del escarpe del Quílamo, delimitado en la parte superior por el Escarpe y en la parte baja por el Rio Jurumbaino, son bloques sub angulosas de cuarcita y areniscas con matriz fina limo arcillosa de color café.

\section{Unidad Upano: (Jurásico)}

Pizarras: pizarras negras moscovíticas altamente meteorizadas, cubren la parte alta del relieve subandino, tienen una potencia que vas desde los 20 hasta los 100 de altura, este tipo de roca subyace sobre bloques de esquistos. Esquistos: en la cordillera Real en el flanco oriental, se observó esquistos negros y cafés, su estructura es de tipo laminar, resultado de la grandes presiones ejercidas sobre las arcillas, que han sufrido una trasformación pasando a ser una roca metamórfica, se encuentras situadas sobre rocas metamórficas tipo pizarras, direccionados por una falla de tipo regional en dirección NS,

Coluviales: Se observaron depósitos coluviales en la parte Oeste del área de estudio, compuestos de bloques de esquistos, que van desde los $10 \mathrm{~cm}$ hasta los $2 \mathrm{~m}$ de espesor en medio de una matriz de roca esquisto meteorizada, se originó por el arrastre de los fragmentos producidos por el levantamiento de la cordillera Real Oriental.

\section{$\underline{\text { Geologia estructural }}$}

Mediante la ortofoto (figura 2) se ubicó la zona de interés, en la que se resalta la parroquia Macas, para una mejor apreciación de las estructuras se sectorizó el área de estudio divididas en cuatro secciones A, B, C y D, digitalizando las estructuras sobres la ortofoto del área total con las estructura.

\section{Perfil estratigráfico}

Desde el occidente hasta el oriente de Macas, se contempla una Unidad, dos formaciones geológicas y los depósitos recientes. La Unidad Upano subyace formando la zona subandina producto de una falla regional inversa, se observó en la zona central del área de estudio la Formación Hollín, en la llanura del Upano se distinguió la Formación Mera y en la cuenca del rio Upano los Depósitos Recientes, se realizó la columna estratigráfica de la parroquia Macas (figura4), donde se explica el tipo de roca que contiene cada unidad y sus formaciones. 
Figura 4. Columna Estratigráfica de la parroquia Macas.

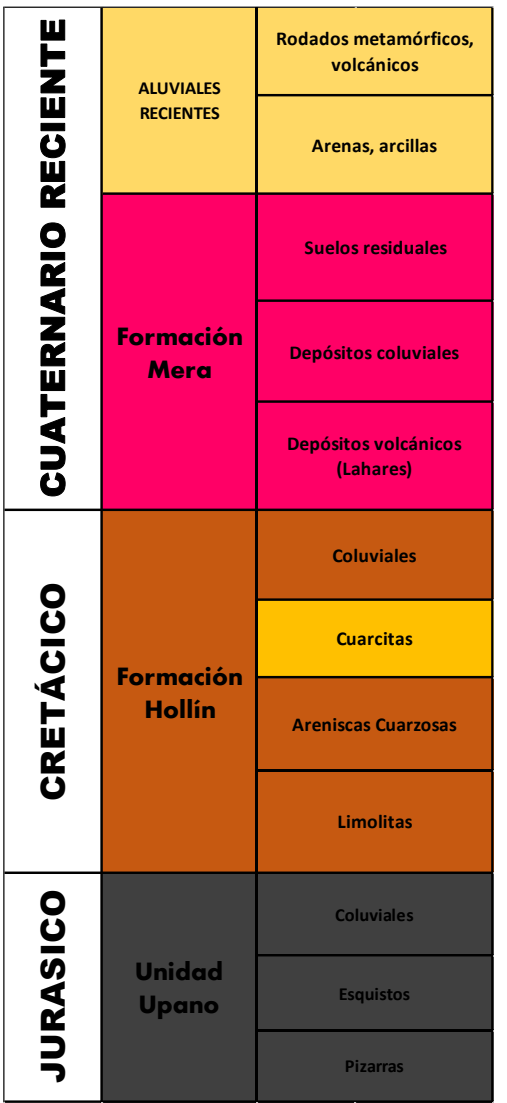

\section{Conclusiones.}

- El área de estudio se encuentra constituida por dos formaciones geológicas y una unidad geológica, como son la Formación Mera y Formación Hollín, y la unidad Upano. Litológicamente el área de estudio está compuesta por depósitos volcánicos y coluviales pertenecientes a la formación Mera; cuarcitas, areniscas cuarzosas y limolitas de la Formación Hollín; esquistos negros, cafés y pizarras negras de la Unidad Upano.

- Geomorfológicamente tenemos cinco tipos de estructuras las cuales son: (1) Rift del Upano, que se formó por fuerzas convergentes extensionales produciendo la rotura de la corteza terrestre creando lo que hoy conocemos como la zona del rio Upano; (2) Meseta Volcano-sedimentaria, ubicada al este del área de estudio, constituida de depósitos de lahares provenientes del volcán Sangay y clastos coluviales del valle del Upano; (3) Valles Fluviales ubicadas en la parte central del área de estudio, formados por los ríos existentes en el área dando un relieve característico en forma "V"; (4) Escarpes de Falla ubicados en la zona del Quílamo, se puede observar en la zona de estudio grandes elevaciones con pendientes pronunciadas que denotan las fracturación de la corteza; (5) Relieves Subandinos ubicados al oeste del área de estudio, formados por el levantamiento de la cordillera real. 
- Estructuralmente se puede apreciar tres fallas inversas importantes que atraviesan el área de estudio en dirección N-S, siguiendo la alineación del flanco oriental de la cordillera real, también se aprecian fallas menores que cortan perpendicularmente a estas fallas principales por lo que enmarcan la zona norte (Proaño, Jiimbitono, y San Isidro) de la parroquia Macas como la idonea para la expansión urbana.

\section{Referencias bibliográficas.}

- Baby, P., Rivadeneira, M., \& Barragán, R. (2004). Cuenca oriente geología y petróleo. Quito.

- Baldock, J. (1982). Geología del Ecuador. División de investigación geologico minera. Ecuador.

- Barnes, J. W., \& Lisle, R. J. (2004). Basic Geological Mapping (Vol. 4). Chichester, England.

- Delagado, J., Padilla, F., \& Barrientos, V. (2010). Prácticas de GEOLOGÍA. Mapas geológicos y Problemas. Universidad De Coruña, La Coruña.

- Echeveste, H. (2016). Manual de levantamiento geológico. Universidad de Buenos Aires, Buenos Aires.

- GAD del canton Morona. (2014). Plan Cantonal de Desarrolo y Ordenamiento Territorial. Macas.

- Hills, E. (1970). Elementos De Geología Estructural. Londres.

- INPRES, I. N. (2005). Fallas Geológicas. San Juan - Argentina.

- Litherland. (1994). The metamorphic belts of Ecuador. Ecuador.

- Moreno Murillo, J., Sánches Aguilar, J., \& Gómez, C. (2017). Guía de Campo I Bogotá.

- Patrice Baby, M. R. (2004). Cuenca oriente geología y petróleo. Quito.

- Weitz, C. (2011). Contactos geológicos/estratigrafía. Recuperado el 28 de 9 de 2017, de https://www.uahirise.org/es/temas/geologic.php

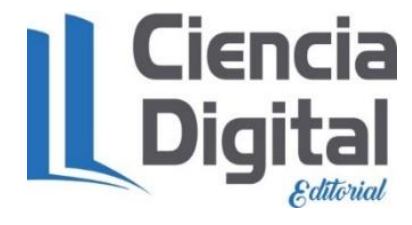




\section{PARA CITAR EL ARTÍCULO INDEXADO.}

Pasato Jarro, J., Cuesta Andrade, G., Nathanael Toapanta., S, \& Granja Carrera, J. (2019). Análisis geológico, estructural y estratigráfico de la ciudad de Macas para la planificación de las zonas de expansión urbana. Ciencia Digital, 3(3.1), 285-294. https://doi.org/10.33262/cienciadigital.v3i3.1.701

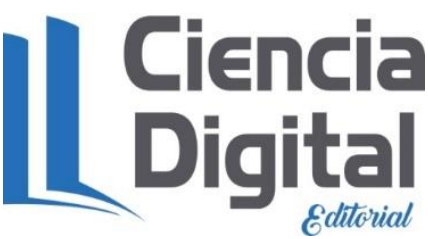

El artículo que se publica es de exclusiva responsabilidad de los autores y no necesariamente reflejan el pensamiento de la Revista Ciencia Digital.

El artículo queda en propiedad de la revista y, por tanto, su publicación parcial y/o total en otro medio tiene que ser autorizado por el director de la Revista Ciencia Digital.
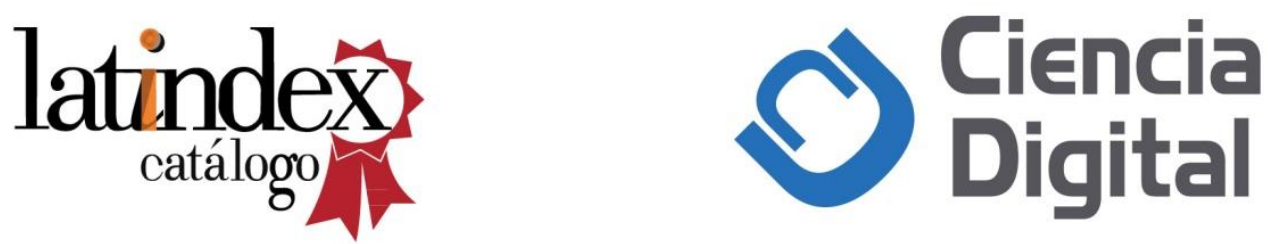